\title{
Cheilitis Granulomatosa - An Uncommon Clinicopathological Entity: A Case Report
}

\author{
Saurabh Goel ${ }^{1 *}$, Manisha Khorate ${ }^{2}$, Prashant Nahar ${ }^{3}$ and Junaid Ahmed ${ }^{4}$ \\ ${ }^{1}$ Post-Graduate Student, Department Of Oral Medicine \& Radiology, Pacific Dental College \& Hospital, Udaipur, Rajasthan \\ ${ }^{2}$ Senior Lecturer, Department of Oral Medicine \& Radiology, Pacific Dental College \& Hospital, Udaipur, Rajasthan \\ ${ }^{3}$ Reader, Department of Oral Medicine \& Radiology, Pacific Dental College \& Hospital, Udaipur, Rajasthan \\ ${ }^{4}$ Professor \& Head of the Department, Department Of Oral Medicine \& Radiology, Pacific Dental College \& Hospital, Udaipur, Rajasthan
}

\begin{abstract}
Granulomatous lesions of the Oral and Orophrayngeal submucosal tissues frequently affecting buccal and labial tissues are uncommon and present a diagnostic dilemma because of the wide variety of possible etiologic factors. The lesion affecting lips initially described by the German dermatologist Miesher as Cheilitis Granulomatosa is a rare disorder characterized by non-remissive enlargement of one or both lips.

The multiple causes and clinical features of Cheilitis Granulomatosa often create a confusing maze through which the clinician must carefully proceed in order to develop an accurate diagnosis and provide an effective treatment. Management considerations for these patients depend upon the results of the investigations, patient's esthetic considerations and severity of the condition.

This article, besides discussing a successfully treated case of Cheilitis Granulomatosa along with gingival involvement in a 27 year old Indian woman, also highlights the importance of differentiating this condition from other lip swellings.
\end{abstract}

Keywords: Cheilitis granulomatosa; Systemic steroids; Orofacial granulomatosis

\section{Introduction}

Cheilitis Granulomatosa (CG) is a rare granulomatous disorder of unknown origin, initially described by German dermatologist Miescher in 1945 as a distinct clinicopathological entity, characterized clinically by diffuse, non tender, soft to firm swelling of one or both lips. CG has often been associated with other Orofacial Granulomatous disorders e.g. Sarcoidosis, Crohn's disease, Atypical Tuberculosis, Anderson-Fabry disease and Allergic reaction. It is also considered as an oligo symptomatic or monosymptomatic form of Melkersson Rosenthal syndrome (MRS).

CG usually affects young adults, mostly in the $2^{\text {nd }}$ decade of life with a female predilection (Ceena et al., 2006). The estimated incidence of CG is $0.08 \%$ in the population (El-Hakim and Chauvin, 2004). The degree of lip swelling can be considerable and is frequently associated with both vertical fissuring and angular cheilitis. In some cases the labial swelling is associated with herpitiform eruptions (Zecha et al., 1976).

Orofacial granulomatosis is a more general term introduced by Wiesenfeld in 1985, which refers to presence of granulomatous inflammation in the oral and facial regions. The oral mucosa is thickened \& edematous with the buccal and labial (70\%) mucosa assuming a corrugated or lobulated appearance. Occasionally, palatal involvement has the appearance of papillary hyperplasia. A characteristic gingival hyperplasia when present has a patchy distribution $\&$ a predilection for anterior region (Sholapurkar et al., 2006).

\section{Case Report}

A 27 year old Indian female patient reported to the Department of Oral Medicine with a chief complaint of swelling in upper lip and anterior gums since last 5 months, which was painless and persistent. It appeared during the $2^{\text {nd }}$ trimester of her pregnancy without any remission even after 1 month of parturition and slowly progressed to the present extent. The patient gave history of anterior gums swelling followed by upper lip swelling and the lip was everted and exposed the vestibular mucosa. There was no apparent history of trauma, allergy to any substance, insect bite, pain in the teeth, pus discharge, fever, facial paralysis or any other history of systemic ailment.

Patient consulted a physician 2 months prior to his visit for the same problem, and was prescribed symptomatic systemic medication, but got no relief. She was unclear what medication was given and did not carry any written prescriptions with her. The family history and past dental history of the patient were nonsignificant. She had no deleterious or parafunctional oral habits.

Extraoral examination revealed a diffuse swelling of upper lip causing fullness in the pre-maxillary region with smooth and intact overlying skin, having ill defined margins (Figure 1). Palpation revealed a normal temperature, nontender enlargement which was soft in consistency.

Intraoral examination revealed cracked upper lip with crustations (Figure 1). In upper anterior region, the gingiva was lobulated and enlarged, extending mesio-distally from 13 to 23 regions and superioinferiorly from labial vestibule to partially covering the crowns with blunting and enlargement of interdental papillae extending palatally

*Corresponding author: Dr. Saurabh Goel, Post-Graduate Student, Department Of Oral Medicine \& Radiology, Pacific Dental College \& Hospital, Airport road, Debari (313024), Udaipur, Rajasthan, Tel: 9982269698; Fax: 0294-2491508; E-mail: drsaurabh_2008@yahoo.com

Received April 29, 2010; Accepted June 03, 2010; Published June 03, 2010

Citation: Goel S, Khorate M, Nahar P, Ahmed J (2010) Cheilitis Granulomatosa - An Uncommon Clinicopathological Entity: A Case Report. J Cancer Sci Ther 2: 086-088. doi:10.4172/1948-5956.1000029

Copyright: (C) 2010 Goel S, et al. This is an open-access article distributed unde the terms of the Creative Commons Attribution License, which permits unrestricted use, distribution, and reproduction in any medium, provided the original author and source are credited. 
in the region of 11 and 21 . In the lower anterior region, gingiva was enlarged in relation to 31,32 , and 33 with gingival recession in relation to 41 with apparent inflammation. Enlarged gingiva was reddish pink in color, soft to firm, with loss of stippling \& bleeding on probing (Figure 1). The dorsum of the tongue showed normal surface papillae with fissuring.

The chief complaint, history and clinical examination led to a provisional diagnosis of Chronic Idiopathic Granulomatous lesion of the upper lip and gingiva.

The differential diagnosis included Cheilitis Granulomatosa, Angioedema, Cheilitis glandularis, Neurofibroma, Exfoliative cheilitis, Plasma cell cheilitis, Sarcoidosis, Crohn's disease, Tuberculosis, Hemangioma, Lymphangioma, Anderson-Fabry disease and Leukemic infiltrate.

Vitality test in relation to 11-23 and 31-42 regions was performed and the respective teeth were found to be vital. Blood investigations (Hb\%, BT, CT, ESR, TLC, DLC), RBS, HbSAg and HIV were in the normal range. Intraoral periapical radiographs in relation to 11-21 and 3142 region revealed mild angular bone loss with intact lamina dura with normal periodontal ligament (PDL) space width (Figure 2) and Orthopantomograph revealed generalized mild angular bone loss with no gross bony pathology. In vitro Hypersensitivity test was negative. An Incisional biopsy of the lip and gingiva was performed under local anaesthesia.

Biopsy specimen revealed stratified squamous parakeratinized epithelium overlying an edematous connective tissue stroma with delicate to dense collagen fibres and few ill-defined areas of

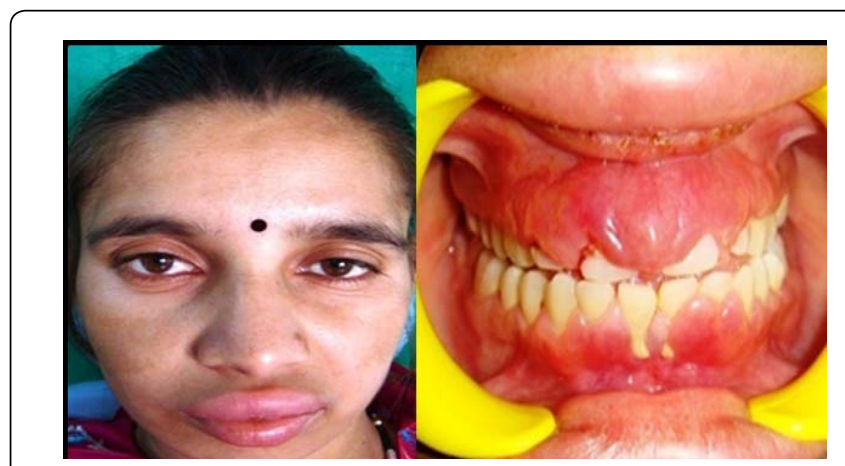

Figure 1: Pre operative photograph showing swelling of upper lip and gingiva.

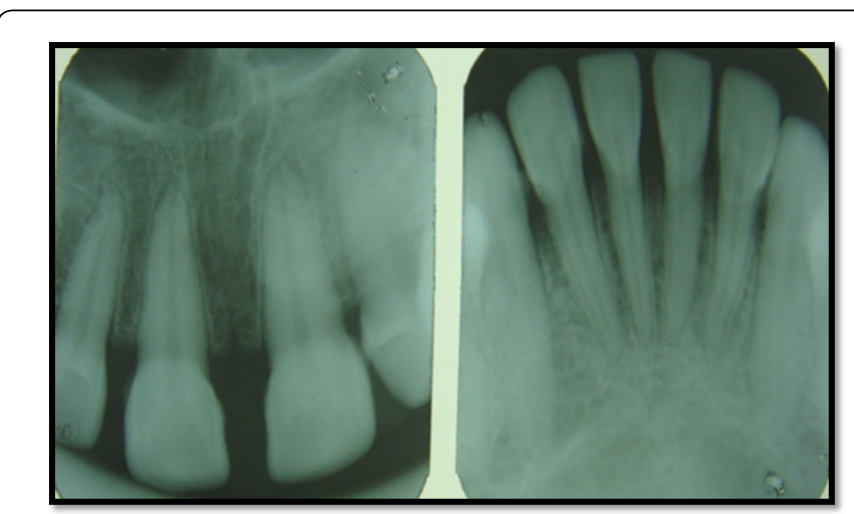

Figure 2: Intraoral periapical radiographs in relation to 11-21 and 31-42 region revealed mild angular bone loss with intact lamina dura with normal periodontal ligament space width.

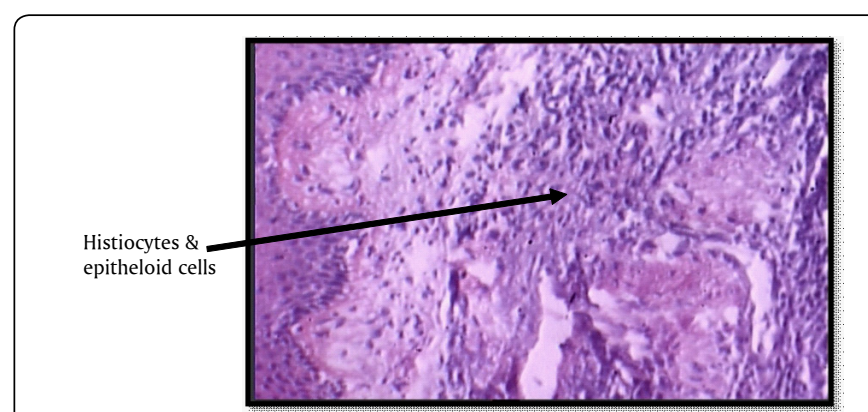

Figure 3: Photomicrograph showing noncaseating granulomatous inflammation with multinucleated giant cells. H\& E. 10x.

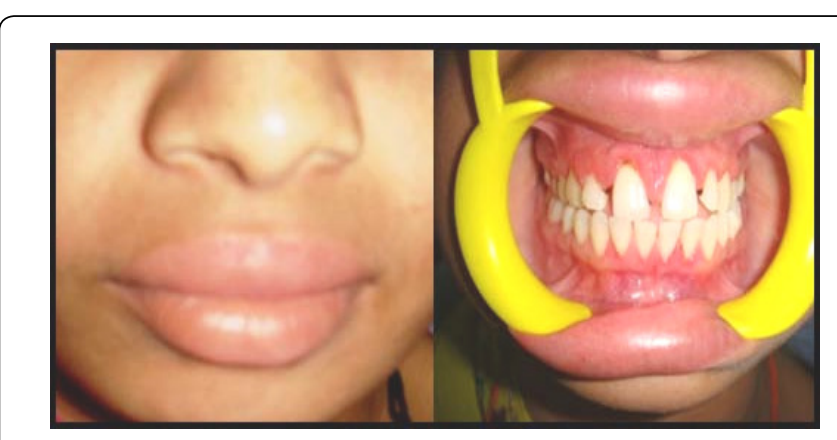

Figure 4: Post operative photograph showing healthy lip and gingiva after 3 months.

noncaseating granulomatous infiltrate consisting of lymphocytes, foamy histiocytes, epitheloid cells and Langerhan's type of multinucleated giant cells (Figure 3), which were suggestive of chronic granulomatous lesion of the lip.

The histopathological findings of the gingival tissue revealed the aforementioned features along with superficial connective tissue showing edematous areas and few areas of thick collagen fibres which was suggestive of a granulomatous lesion.

The chief complaint, history, clinical examination and subsequent investigations led us in arriving at a final diagnosis of Cheilitis granulomatosa of the upper lip.

Treatment given to the patient consisted of Systemic Prednisolone $10 \mathrm{mg}$ tid for 7 days. Patient reported after 1 week with significant reduction in signs $\&$ symptoms of lip swelling. Same treatment was continued for another 14 days with follow up every 1 week. After $3^{\text {rd }}$ week of commencement of treatment, the dose of prednisolone was tapered down gradually and finally withdrawn after 3 months (Figure 4).

Gingiva did not show any remarkable response to systemic glucocorticoid therapy, hence surgical treatment of gingivoplasty was advised (Figure 4).

\section{Discussion}

The clinical presentation of CG is highly variable. The etiology \& pathogenesis of CG is unknown. Hornstein suggested that the disorder is polyetiologic and may be caused by an alteration in autonomic nervous system function localized to facial skin, resulting in increased vascular permeability and edema. Chronic infectious foci, food sensitivities and obstruction of lymphatic vessels have all been suggested as contributing factors (Ceena et al., 2006). 


\begin{tabular}{|l|l|}
\hline Diagnostic considerations & Features not consistent with Cheilitis granulomatosa \\
\hline Angioedema & Swelling resolves in 24-48 hrs and recurs again on antigenic stimulation. \\
\hline Cheilitis glandularis & Inflamed orifices of secretary ducts with red macules on mucosa. \\
\hline Neurofibroma & Slowly progressive enlargement; pathognomonic and histology. \\
\hline Exfoliative cheilitis & It is precipitated by lip biting, trauma, along with the presence of epithelial tags. \\
\hline Plasma cell cheilitis & The classic clinical appearance is a flat-to-slightly raised, eroded plaque or patch, usually on the lower lip of elderly patient. \\
\hline Sarcoidosis & Characteristic skin lesions; mediastinal involvement; lungs and liver involvement. \\
\hline Crohn's disease & Gastrointestinal signs and symptoms; oral ulcerations and fissures. \\
\hline Tuberculosis & Past history of tuberculosis, and other clinical features of TB. \\
\hline Hemangioma & Lesions congenital; vascular proliferation. \\
\hline Lymphangioma & Congenital lesion and characteristic appearance. \\
\hline Anderson-Fabry disease & Characteristic angiokeratomas of skin; history of pain affecting extremities. \\
\hline Leukemic infiltrate & Histologic features of atypical infiltrate; abnormal peripheral WBCs. \\
\hline
\end{tabular}

Table 1: Differential diagnostic possibility for patients with lip swelling (Sholapurkar et al., 2006; Grave et al., 2009; Rogers and Bekic, 1997).

Worsaae and Pindborg in their cases noted that gingival swelling may precede the lip swelling and the gingival changes may appear either as diffuse edematous or as distinct small, irregular, bluish red edematous swelling on the gingival surface. Further they have reported that gingival manifestations appeared mainly in the anterior part of the mouth (Worsae and Pindborg, 1980). In the present case gingival enlargement preceded the lip swelling. Diffuse enlargements of the interproximal and marginal gingiva in both maxillary and mandibular anterior region were also seen. In our case CG occurred on the upper lip which was in accordance to the available literature which stated that upper lip was the most common site of occurrence (Worsae and Pindborg, 1980).

Histologically, within the lamina propria, dilation of lymphatic vessels, perivascular lymphocytic infiltration and to a varying degree non necrotizing granulomas are seen. Typically, the granulomas appear to cluster around scattered vessels and are not well formed or discrete. Fibrosis may be present in long term lesions (Ceena et al., 2006).

Diagnosis of CG is done by ruling out other conditions. The clinical differential diagnosis for a swollen lip can be extensive but a good history and careful clinical examination will usually eliminate many diagnostic possibilities. Ultimately the diagnosis is made by correlation of clinical data and the histopathologic findings. Table 1 shows various differential diagnosis possibilities for patients with lip swellings, and their characteristic features.

Management of CG is dependent on accurate diagnosis of the condition and identification of any precipitating factors. Patients without dental infections who present with clinical features suggestive of CG should be questioned regarding the presence of systemic signs and symptoms of crohn's disease, sarcoidosis or a history of angioedema. In the presence of positive findings, the patient should be referred for appropriate medical evaluation.

Management of CG is difficult due to its unknown etiology. Unfortunately, no therapy has provided predictable results thus far. The goal of therapy is focussed on reducing the swelling to an acceptable state for the patient (El-Hakim and Chauvin, 2004).

Various treatment modalities such as surgery, drug therapy with antituberculous drugs, antibiotics, vitamins, Phenylbutazone, ACTH, oral and intralesional corticosteroids and boiled water injections have been used in the treatment of CG (Ceena et al., 2006).

Corticosteroids have been shown to be effective in reducing lip swelling and preventing recurrences and are considered the mainstay of therapy. Patient with mild swelling were commenced on topical steroids. More pronounced swelling of the lip or deterioration of lip swelling was treated with intralesional triamcinolone acetonide $0.1 \%$ injections. Patients with more extensive lip swelling were initially treated with systemic medication (Grave et al., 2009). Surgery was performed only in severely disfiguring cheilitis and once the disease had been brought into a quiescent phase. The criteria for choice of treatment would seem to be subjective with little scientific basis for selecting one treatment protocol over another (Grave et al., 2009).

In our case the patient had an extensive lip swelling; hence it was initially treated with systemic glucocorticoid i.e prednisolone for three months with regular follow up and patient got complete regression in signs and symptoms after 3 months.

CG remains an enigmatic disorder with multiple causes. The dental practitioner is likely to encounter patients with this disorder and a proper knowledge of this idiopathic condition is imperative and places a major role in its successful diagnosis and treatment.

\section{References}

1. Ceena DE, Ashok L, Shivprasad S, Anitha B, Ahmed Mujib BR (2006) Cheilitis granulomatosa. JIAOMR 18: 167-169.

2. El-Hakim M, Chauvin P (2004) Orofacial granulomatosis presenting as persistent lip swelling: review of six new cases. J Oral Maxillofac Surg 62: 1114 1117.

3. Grave B, McCullough M, Wiesenfeld D (2009) Orofacial granulomatosis--a 20 year reviews. Oral Dis 15: 46-51.

4. Rogers RS, Bekic M (1997) Diseases of the lips. Semin Cutan Med Surg 16 328-336.

5. Sholapurkar AA, Vineetha R, Pai KM, Suhas S, Bhat SS (2006) Cheilitis granulomatosa- Report of two cases with clinical and diagnostic implications. JIAOMR 18: 218-223.

6. Worsae N, Pindborg JJ (1980) Granulomatous gingival manifestations of Melkersson-Rosenthal Syndrome. Oral Surg Oral Med Oral Pathol 49: 131138.

7. Zecha JJ, Van DijK L, Hadders HN (1976) Cheilitis granulomatosa (MelkerssonRosenthal syndrome). Oral Surg Oral Med Oral Pathol 42: 454-460. 